\title{
Micro-particle deposition and lobar distribution of mass flow in human upper respiratory tract model
}

\author{
HUANG JianHua ${ }^{1} \&$ ZHANG LianZhong ${ }^{1,2 *}$ \\ ${ }^{1}$ School of Physics, Nankai University, Tianjin 300071, China; \\ ${ }^{2}$ State Key Laboratory of Loess and Quaternary Geology, Institute of Earth Environment, Chinese Academy of Sciences, Xi'an 710075, China
}

Received June 1, 2010; accepted July 22, 2010

\begin{abstract}
A representative human upper respiratory tract model consisting of oropharyngeal and asymmetric tracheobronchial (TB) airways from the trachea $(\mathrm{G} 0)$ to the main lobar bronchi $(\mathrm{G} 2)$ was constructed. Laminar-to-turbulent airflow for typical inhalation modes as well as micro-particle transportation, wall deposition and mass flow to lobes was simulated using the CFX10.0 software from Ansys Inc. The numerical particle deposition efficiency of the oropharynx region and two generations (G1 and G2) of TB airways shows great agreement with the experimental data obtained from realistic casts. The particle deposition pattern indicates that inertial impaction is the primary mechanism in the human upper airway, and turbulence dispersion performs crescent influence especially for small particles. The initial positions of particles with different fates are confined to specifically concentrated zones. The particle mass distributions of five lobes are close to airflow distributions. The upper lobes receive fewer particles than lower lobes and the right middle lobe receives the least.
\end{abstract}

upper respiratory tract, asymmetric tracheobronchial airways, transportation and deposition, lobar differences, computational fluid dynamics simulation

Citation: Huang J H, Zhang L Z. Micro-particle deposition and lobar distribution of mass flow in human upper respiratory tract model. Chinese Sci Bull, 2011, 56: 380-385, doi: 10.1007/s11434-010-4305-y

Inhaled particle deposition in the human respiratory tract is a concern in lung diseases. Accurate investigation of aerosol deposition in the human upper respiratory tract (URT) and lobar distribution are important for the analysis of "filtering effects" of human URT and correct air/particle inlet conditions to the deeper bronchial airways. The human upper respiratory tract consists of the oral cavity, nasal cavity, pharynx/larynx airway and tracheobronchial (TB) airways. Numerous investigations have been carried out in experimental or numerical methods on one part or several parts of the human respiratory tract.

Previous particle deposition investigations in the oropharyngeal airway have used realistic replicas from cadavers [1], idealized oral models [2] and idealized mouth-throat models $[3,4]$. In TB airway studies, the effective methods

*Corresponding author (email: zhanglz@ nankai.edu.cn) include theoretical predictions on symmetrical bifurcation [5], experimental measurements on realistic replicas [6-8], Monte Carlo stochastic simulations $[9,10]$ and computational fluid dynamics (CFD) determinate simulations [11,12].

Most studies have focused on one part or several separated parts of human URT, but a few studies investigate the particle deposition in the whole region of human URT [13] or predict the lobar distribution of air and particle mass flow into the five lobes. Whole region URT studies avoid any assumptions of boundary conditions between separate parts. A more precise human URT model will improve the accuracy of prediction.

In this study, we construct a representative human URT model with oropharyngeal and asymmetric TB airways, as well as the refined unstructured mesh. The particle deposition efficiency of the oropharyngeal region and two generations of TB airways are compared with theoretical models, 
experimental data obtained from realistic casts under three inspiratory rates $(15 \mathrm{~L} / \mathrm{min}, 30 \mathrm{~L} / \mathrm{min}$ and $60 \mathrm{~L} / \mathrm{min})$. The lobar differences of airflow and particle mass flow are investigated for the first time using the CFD simulation method. The local deposition pattern and corresponding released position at mouth inlet are also studied.

\section{Numerical model}

\subsection{Human upper airway model}

The present human URT model consists of two parts: oropharynx and asymmetric TB airways, as shown in Figure 1. The oropharynx airway was constructed from a human cast [1], including oral cavity (mouth), pharynx, larynx and trachea (G0) as well as the variations described by Zhang et al. [2]. The dimensions of the asymmetric TB airways were adopted from "the typical path lung model" described by Yeh et al. [14]. The "typical path lung model" considered the human lungs as five parts, i.e. three right lobes and two left lobes. There are two generations in the present TB airways. The first generation (G1) contains the first bifurcation and two main lung bronchial tubes leading to the right and left lungs. There are two regions in the second generation (G2). "G21" represents the right lung, consisting of two bifurcations and three main lobes of bronchi leading to the upper, middle and lower lobes of the right lung. "G22" represents the left lung, containing one bifurcation and two main lobes of bronchi leading to the upper and lower lobes of the left lung. The airway segment diameters, lengths, branching angles were employed to construct the asymmetric bronchial bifurcations; the angles of inclination to gravity were ignored in this study. For the seamless join of the oropharyngeal and TB airways, the TB airway segment diameters and lengths are scaled-down. The detailed data of the model geometry are shown in Table 1.

\subsection{Transport equations}

(i) Airflow. The viscous, isothermal and incompressible flows in human URT, the Reynolds Averaged NavierStokes (RANS) equations are modeled by employing the eddy viscosity and eddy diffusivity hypotheses. These hypotheses assumed that the Reynolds stresses in RANS equations can be related to the mean velocity gradients and turbulent viscosity. Therefore, the governing equations for the constant inspiratory airflow include the conservation of mass

$$
\nabla \cdot \overline{\boldsymbol{u}}=0
$$

and momentum

$$
\frac{\partial \overline{\boldsymbol{u}}}{\partial t}+\nabla \cdot(\overline{\boldsymbol{u}} \otimes \overline{\boldsymbol{u}})=-\frac{1}{\rho} \nabla p^{\prime}+\nabla \cdot\left(v_{\mathrm{eff}}\left(\nabla \overline{\boldsymbol{u}}+(\nabla \overline{\boldsymbol{u}})^{\mathrm{T}}\right)\right),
$$

where $\overline{\boldsymbol{u}}$ is the averaged fluid velocity vector, $\rho$ is the fluid

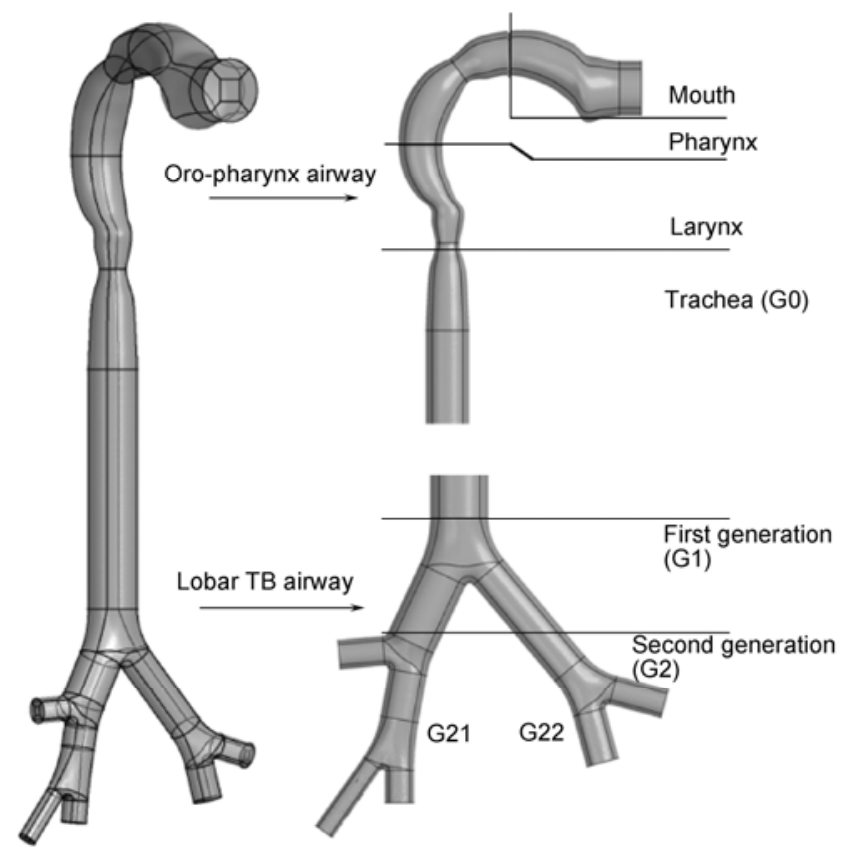

Figure 1 Schematic of the human upper respiratory tract model.

density, and $p^{\prime}$ is modified pressure (which is assumed equal to pressure in the default solver of ANSYS-CFX). $v_{\text {eff }}$ is the effective kinetic viscosity, defined as $v_{\text {eff }}=v+v_{\mathrm{t}}$, where $v$ is kinetic viscosity and $v_{\mathrm{t}}$ is turbulence kinetic viscosity linked to the turbulence kinetic energy $k$ and turbulent frequency $\omega$ via the relation, $v_{\mathrm{t}}=k / \omega$ [15]. The Wilcox $k-\omega$ turbulence model was adopted to simulate the airflow field in the URT model.

Turbulence kinetic energy $(k)$ equation:

$$
\frac{\partial k}{\partial t}+\nabla \cdot(\boldsymbol{u} k)=\nabla \cdot\left[\left(v+\frac{v_{\mathrm{t}}}{\sigma_{k}}\right) \nabla k\right]+P_{k}-\beta^{\prime} k \omega ;
$$

Pseudo-vorticity $(\omega)$ equation:

$$
\frac{\partial \omega}{\partial t}+\nabla \cdot(\boldsymbol{u} \omega)=\nabla \cdot\left[\left(v+\frac{v_{\mathrm{t}}}{\sigma_{\omega}}\right) \nabla \omega\right]+\alpha \frac{\omega}{k} P_{k}-\beta \omega^{2} .
$$

$P_{k}=v_{\mathrm{t}} \nabla \boldsymbol{u} \cdot\left(\nabla \boldsymbol{u}+(\nabla \boldsymbol{u})^{\mathrm{T}}\right)$ is the turbulence production due to viscous force, and $\beta^{\prime}, \alpha, \beta, \sigma_{k}$ and $\sigma_{\omega}$ are turbulence constants, i.e. $\beta=0.09, \alpha=5 / 9, \beta=0.075, \sigma_{k}=\sigma_{\omega}=2$.

(ii) Particle transport. Micro-particle transport in the URT model is considered to be dilute monodisperse rigid sphere suspensions, with large particle-to-air density ratio and negligible particle rotation. In this case, the drag force is dominant, based on the order-of-magnitude arguments (see [16]). Hence, the particle trajectory equation can be written as

$$
m_{\mathrm{P}} \frac{\mathrm{d} \boldsymbol{u}^{\mathrm{P}}}{\mathrm{d} t}=\frac{1}{8} \pi \rho d_{\mathrm{p}}^{2} C_{D \mathrm{p}}\left|\boldsymbol{u}-\boldsymbol{u}^{\mathrm{P}}\right|\left(\boldsymbol{u}-\boldsymbol{u}^{\mathrm{P}}\right),
$$

where $m_{\mathrm{P}}, \boldsymbol{u}^{\mathrm{P}}, d_{\mathrm{p}}$, are the one spherical particle mass, particle 
Table 1 Dimensions of the TB airways

\begin{tabular}{|c|c|c|c|c|c|c|c|c|}
\hline & \multicolumn{2}{|c|}{ Parent (mm) } & \multicolumn{6}{|c|}{ Daughter (mm) } \\
\hline & $L_{0}$ & $D_{0}$ & $L_{1}$ & $D_{1}$ & $\theta_{1}\left({ }^{\circ}\right)^{\mathrm{a})}$ & $L_{2}$ & $\mathrm{D}_{2}$ & $\theta_{2}\left({ }^{\circ}\right)$ \\
\hline \multicolumn{9}{|c|}{ Trachea } \\
\hline G0 & 100 & 15.8 & & & & & & \\
\hline \multicolumn{9}{|c|}{ 1st generation } \\
\hline G1 & 100 & 15.8 & 24.29 (to G2.1) & 13.76 & 25 & 44.26 (to G2.2) & 10.85 & 40 \\
\hline \multicolumn{9}{|c|}{ 2nd generation } \\
\hline $\mathrm{G} 2.2$ & 44.26 & 10.85 & 11.16 (to G3.3) & 9.04 & 25 & 11.40 (to G3.4) & 8.10 & 35 \\
\hline \multicolumn{9}{|c|}{ 3rd generation } \\
\hline G3.1 & 9.60 & 8.02 & 6.29 & 5.97 & 28 & 6.29 & 5.97 & 28 \\
\hline G3.2 & 23.74 & 10.45 & 17.84 (to G4.1) & 5.66 & 25 & 6.92 (to G4.2) & 7.94 & 5 \\
\hline G3.3 & 11.16 & 9.04 & 10.45 & 7.11 & 48 & 10.45 & 7.11 & 48 \\
\hline G3.4 & 11.40 & 8.10 & 8.49 & 6.56 & 30 & 8.49 & 6.56 & 30 \\
\hline \multicolumn{9}{|c|}{4 th generation } \\
\hline G4.2 & 6.92 & 7.94 & 8.57 & 6.29 & 23 & 8.57 & 6.29 & 23 \\
\hline
\end{tabular}

a) $\theta$ is the branching angle.

velocity vector and particle density, respectively. $C_{D \mathrm{p}}$ is the drag force coefficient given as

$$
C_{D \mathrm{p}}=C_{D} / C_{\mathrm{slip}}
$$

where

$$
C_{D}=\max \left(\frac{24}{\operatorname{Re}_{\mathrm{P}}}\left(1+0.15 R e_{\mathrm{P}}^{0.687}\right), 0.44\right)
$$

with particle Reynolds number, $R e_{\mathrm{p}}=\left|\boldsymbol{u}-\boldsymbol{u}_{\mathrm{p}}\right| d_{\mathrm{p}} / v$, and $C_{\text {slip, a }}$ correlation for the Cunningham correction (i.e. slip) factor.

The boundary conditions of airflow are set to uniform velocity profiles at the mouth inlet, and zero relative pressure at the five lobar tube outlets; 100000 particles are released from the mouth inlet with uniform distribution. The initial particle velocity is set equal to that of the fluid and one-way coupling was assumed between the air and particle flow fields.

The unstructured mesh topology of the URT model was generated by ICEM-CFD (Ansys Inc., Canonsburg, PA). The refined unstructured mesh contains approximately 850000 nodes and 3000000 elements, which has 2000000 tetrahedrons and 1000000 prisms composed by multiple boundary layers of flat prismatic elements adopted to provide enhanced resolution of the airflow field near to the no-slip wall. The sensitivity study was performed by increasing the mesh elements to 4100000 and particle number to 200000. All computations were performed on an Intel Pentium $42.8 \mathrm{GHz}$ personal computer with 1.5 GB RAM. When the solution was at stable conditions, the flow field was assumed to have converged when the three dimensionless momentum components residual was less than $10^{-4}$ and the mass residual was less than $10^{-5}$. A typical run time for the fluid flow simulations with serial algorithms was approximately $12 \mathrm{~h}$. When using the converged flow field solution, the particle trajectory simulations required ap- proximately $1 \mathrm{~h}$ for each Stokes number considered.

\section{Results and discussion}

\subsection{Particle deposition efficiency}

For direct comparison with the literature, two different definitions on deposition fraction/efficiency and Stokes numbers were adopted. The particle deposition fraction of the oropharynx region is defined as the ratio of number of deposited particles in the oropharynx region to the number of those entering the mouth inlet. The Stokes number in oropharynx region is defined as $S t=\rho_{\mathrm{p}} d_{\mathrm{p}}^{2} U /(9 \mu D)$ [2], where $U$ is mean velocity in the mouth inlet and $D$ is the diameter of mouth inlet. The particle deposition efficiency of the TB airways is defined as the ratio of number of deposited particles in one region to the number of those entering this region. The Stokes number in the TB airways is defined as $S t=\rho_{\mathrm{p}} d_{\mathrm{p}}^{2} U /(18 \mu D)$ [7], where $U_{0}$ is mean velocity through the parent tube, and $D_{0}$ is the diameter of the parent tube.

Figure 2 shows the comparison of the simulated deposition fraction in the oropharynx region with the experimental data [1] and numerical results [2], under three inspiratory flow rates $(15 \mathrm{~L} / \mathrm{min}, 30 \mathrm{~L} / \mathrm{min}, 60 \mathrm{~L} / \mathrm{min})$. The deposition data show great agreement with the experimental data and empirical formulation $\eta=1-\exp (-6.66$ Stk) [1]. The oropharynx region serves as the "entryway" for the TB airways with asymmetric bronchial bifurcations, and thus the exact simulation of the oropharynx region in the present work ensured the accuracy of airflow and particle transportation in the TB airways.

The TB airways in the present model contain two generation bifurcations. The deposition efficiency in two generations is compared with several theoretical models $[1,5,17]$ and experimental data and the results are shown in 


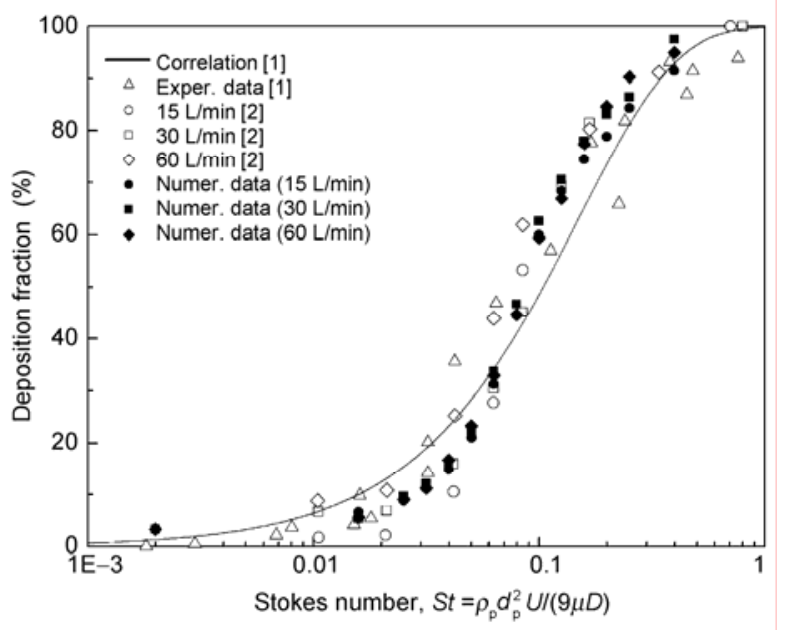

Figure 2 Comparison of simulated particle deposition fraction of oropharynx region with the experimental data and numerical results.

Figure 3. There are two parts in the second generation, with different dimensions of length and diameter, as well as the bifurcate angle. Thus, multiple Stokes numbers and theoretical lines were applied to comparing the deposition efficiencies in this generation (Figure 3(b)). In the first generation, our simulated results are lower than the experimental data $[7,18]$, but have high agreement with the empirical model developed from experimental measurement by Zhou et al. [7] $\eta=1-\exp (-5.39 \cdot \alpha \cdot S t k), \alpha=0.75$. In the second generation, the simulated deposition efficiencies of two parts (G21 and G22) show high agreement with the experimental data and empirical model $\eta=1-\exp \left(-5.39 \cdot \alpha \cdot S t k^{1.34}\right)$, where $\alpha$ is 1.326 and 0.695 for the $\mathrm{G} 21$ and $\mathrm{G} 22$ respectively.

\subsection{Local deposition and particle released position}

Figure 4 illustrates the local deposition pattern in the URT model and corresponding initial position at the mouth inlet for particle sizes of $1 \mu \mathrm{m}$ and $10 \mu \mathrm{m}$, respectively, under the normal inspiratory flow rate $\left(Q_{\mathrm{in}}=30 \mathrm{~L} / \mathrm{min}\right)$. The deposited particles mainly concentrated in the regions with a rapidly changing cross section and geometric structure, such as the lower portion of oral cavity, soft palate, upstream of glottis and carina of bronchial bifurcations. A long and narrow null deposition zone appears in the middle of the concentrated deposition area of the outer bend of the pharynx/larynx, indicating the proper simulation of the uvula in the soft palate. A few particles, in particular the small particles, are deposited in regions with flat geometric structure, such as the trachea and main bronchi tubes because of turbulence dispersion and recirculation flow. Therefore, in the TB airways, the inertial impaction deposition is the primary mechanism, especially for large particles, and turbulence dispersion has a crescent influence on the particle deposition for the small particles. The inlet positions of particles deposited in different regions are apparently confined to specific areas.
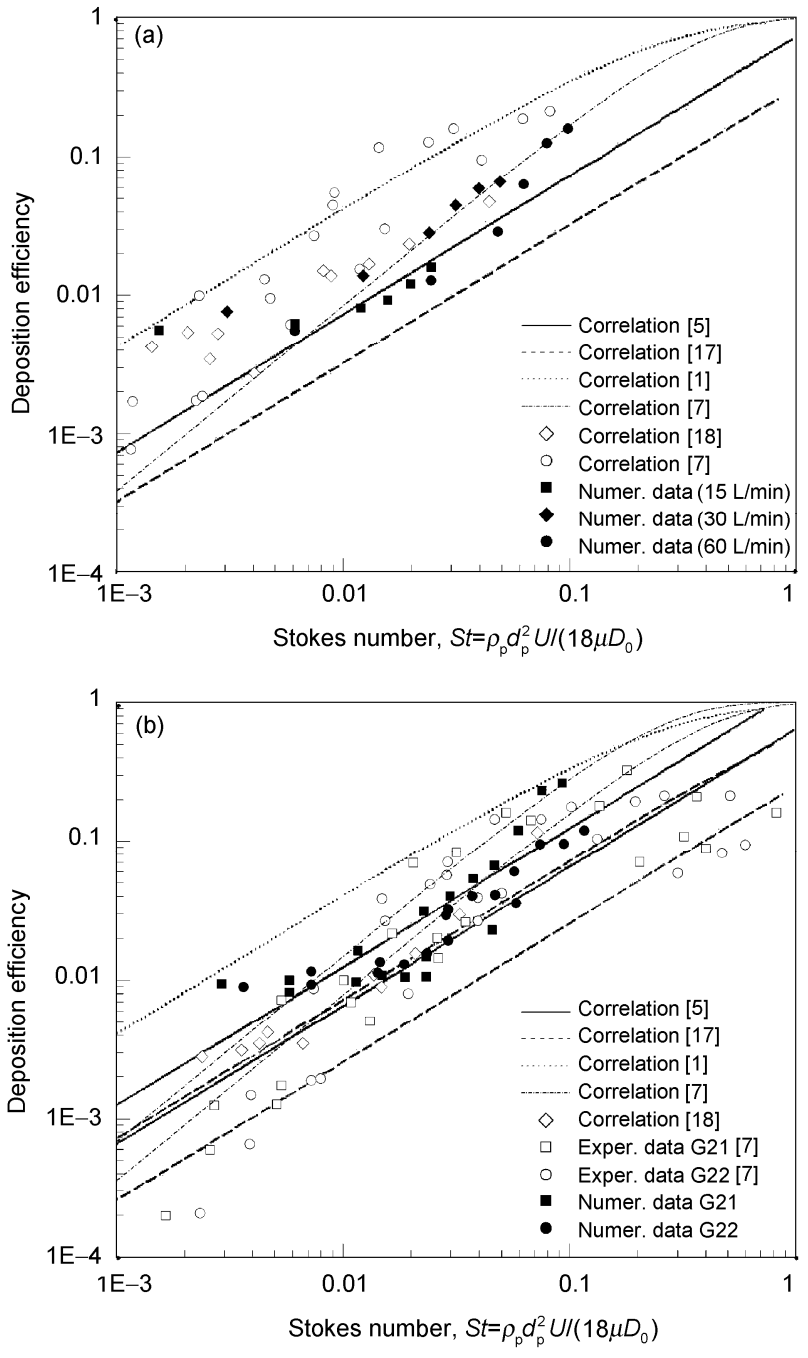

Figure 3 Comparisons of simulated particle deposition efficiency of the first (a) and second (b) generation with the theoretical models and experimental data.

\subsection{Mass flow fraction}

The volume of air entering a lobe of the lung provides the particles available for deposition in deeper generations of that lobe. Different dimensions of length and diameter, as well as the branch angle results in the mass flow ratio of both air and particles transported into the five lobes (i.e. right upper, right middle, right lower, left upper and left lower) being asymmetric. Figure 5 shows the air and particle mass flow fraction (air/particle mass flow entering each lobe divided by the mass flow at the mouth inlet) for three steady inspiratory rates $(15 \mathrm{~L} / \mathrm{min}, 30 \mathrm{~L} / \mathrm{min}$ and $60 \mathrm{~L} / \mathrm{min})$. The air mass flow of five lobes is nearly identical to the previous results of uniform lung expansion [19].

The particle mass flows entering the lower lobes (right lower and left lower) are greater than the upper lobes (right upper and left upper) for all particle diameters, and the right middle lobe receives the least particles. Therefore more particles will deposit in lower lobes, which corresponds with the lobar distribution of particle deposition in the TB 

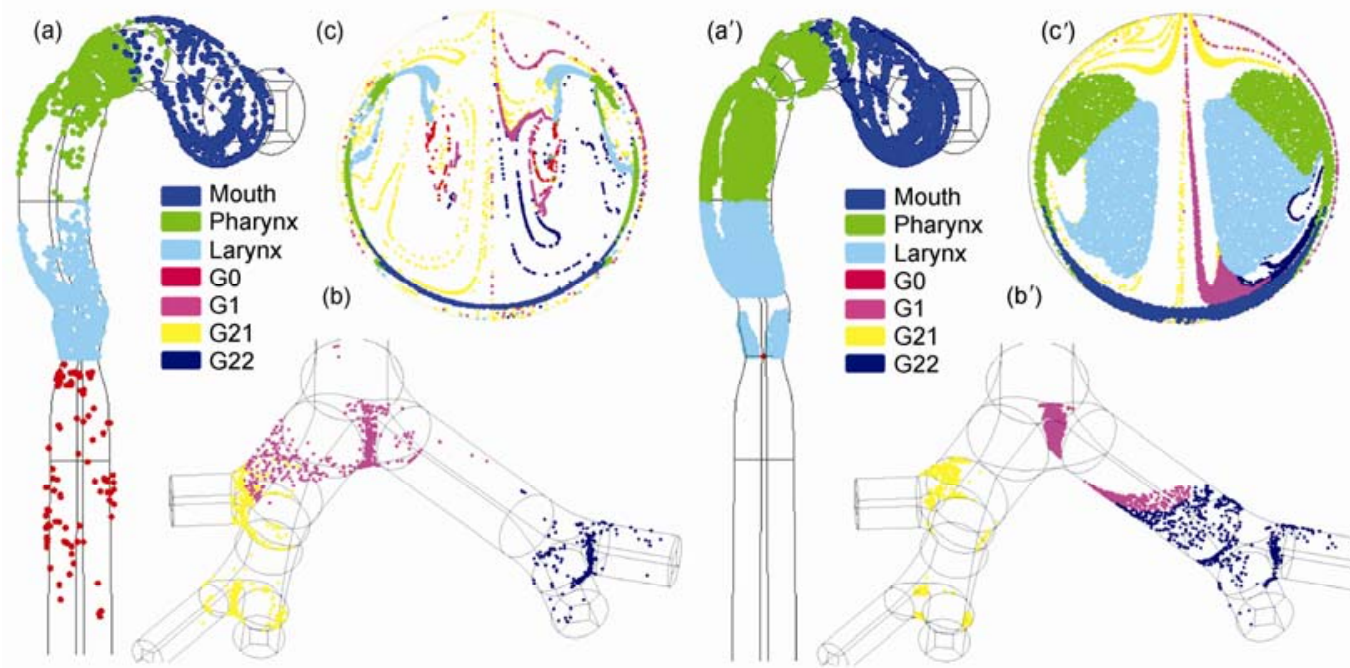

Figure 4 Particle deposited position in the oropharynx region, bronchi region and initial position at the mouth inlet for $Q_{\text {in }}=30 \mathrm{~L} / \mathrm{min}$. (a) $-(\mathrm{c})$ and $\left(\mathrm{a}^{\prime}\right)-\left(\mathrm{c}^{\prime}\right)$ correspond for particle sizes of $1 \mu \mathrm{m}$ and $10 \mu \mathrm{m}$, respectively.
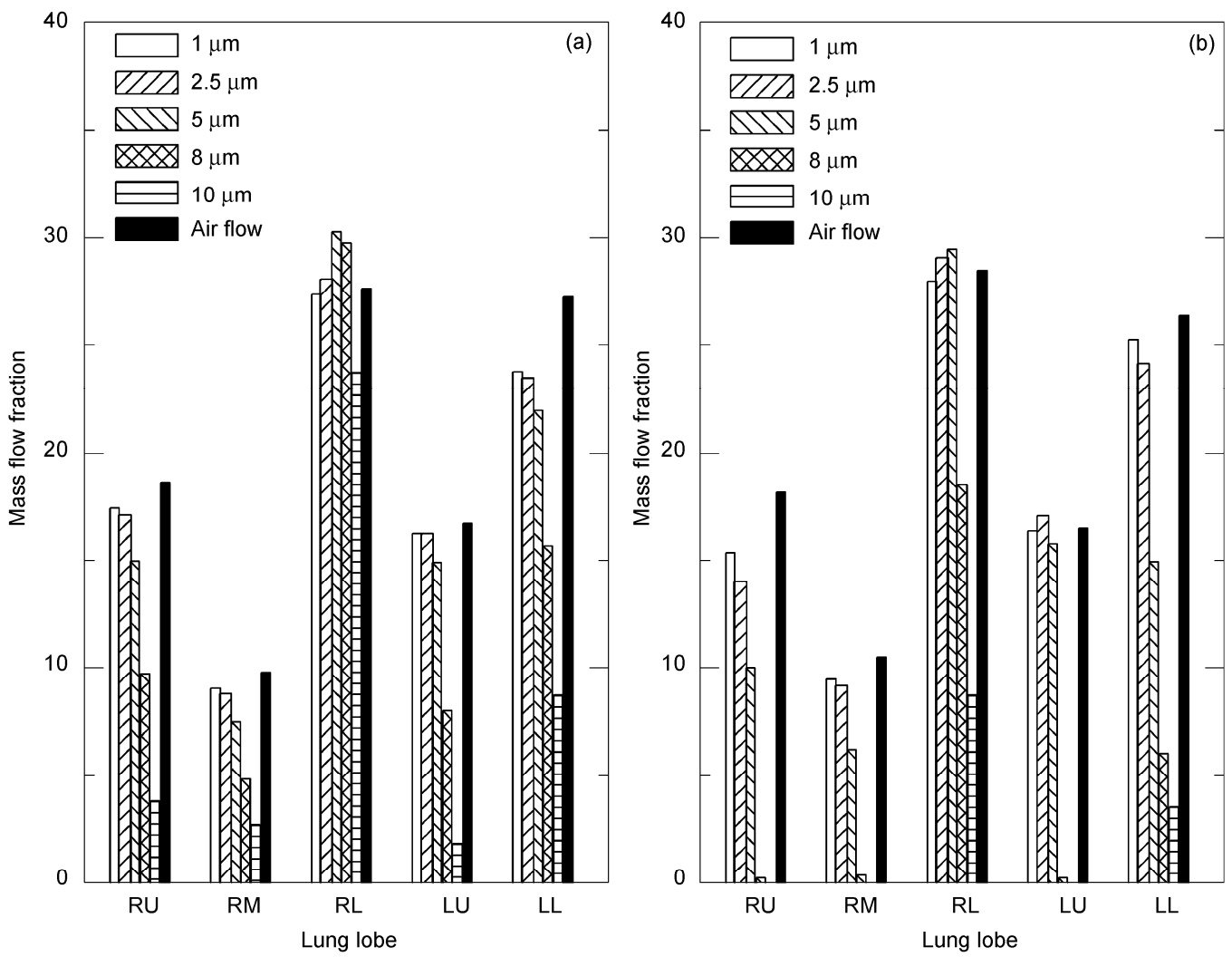

Figure 5 Air and particle mass flow fraction of five lobes for inspiratory rates of $30 \mathrm{~L} / \mathrm{min}$ (a) and $60 \mathrm{~L} / \mathrm{min}$ (b).

airways reported previously [14,20]. The particle mass flow fractions are similar to air mass flow fractions for small particles $(1 \mu \mathrm{m}$ and $2.5 \mu \mathrm{m})$ because of small deposition in the human upper airway, and decreased gradually for large particles (larger than $5 \mu \mathrm{m}$ ) because of the increased impaction deposition in the human upper airway.

\section{Conclusions}

The representative upper airway model with asymmetric TB airways is validated numerically for three inspiratory flow rates and different particle diameters. The particle deposition efficiency in both the oropharynx region and TB air- 
ways has high agreement with theoretical models and experimental data. Thus, the present upper airway model is considered an accurate simulation of the actual human URT.

The inertial impaction is the primary deposition mechanism in both the oropharynx region and primal generations of TB airways, and the turbulence dispersion influence increases gently in the primal generations of the TB airways especially for the small particles. Particles deposited or entering into the lobes are released from specific concentrated zones. According to the correlation between the released and deposited position, the delivery of medical aerosols would be efficient and effective.

The particle mass distributions of the five lobes are similar to the airflow distributions. When compared with the two lower lobes, the two upper lobes receive fewer particles, and the right middle lobe receives the least particles. Based on the accurate simulation of particle deposition in the human upper airway, the lobar distributions of air and particle mass flow will provide accurate boundary conditions for further investigations of the deeper bronchial airways.

This work was supported by the National Natural Science Foundation of China (10672081) and the Foundation of Chinese State Key Laboratory of Loess and Quaternary Geology.

1 Cheng Y S, Zhou Y, Chen B T. Particle deposition in a cast of human oral airways. Aerosol Sci Tech, 1999, 31: 286-300

2 Zhang Z, Kleinstreuer C, Kim C S. Micro-particle transport and deposition in a human oral airway model. J Aerosol Sci, 2002, 33: 1635-1652

3 Grgic B, Finlay W H, Heenan A F. Regional aerosol deposition and flow measurements in an idealized mouth and throat. J Aerosol Sci, 2004, 35: 21-32

4 Johstone A, Uddin M, Pollard A, et al. The flow inside an idealised form of the human extra-thoracic airway. Expt Fluids, 2004, 37: 673-689
5 Cai F S, Yu C P. Inertial and interceptional deposition of spherical particles and fibers in a bifurcating airway. J Aerosol Sci, 1988, 19: 679-688

6 Cohen B S, Susman R G, Lippmann M. Ultrafine particle deposition in a human tracheobronchial cast. Aerosol Sci Tech, 1990, 12: 1082-1091

7 Zhou Y, Cheng Y S. Particle deposition in a cast of human tracheobronchial airways. Aerosol Sci Tech, 2005, 39: 492-500

8 Zhou Y, Su W C, Cheng Y S. Fiber deposition in the tracheobronchial region: Deposition equations. Inhal Toxicol, 2008, 20: 1191-1198

9 Hofmann W, Koblinger L. Monte Carlo modeling of aerosol deposition in human lungs. Part I: Simulation of particle transport in a stochastic lung structure. J Aerosol Sci, 1990, 21: 661-674

10 Sturmand R, Hofmann W. 3D-visualization of particle deposition patterns in the human lung generated by Monte Carlo modeling: Methodology and applications. Comput Biol Med, 2005, 35: 41-56

11 Longest P W, Oldham M J. Mutual enhancements of CFD modeling and experimental data: A case study of $1 \mu \mathrm{m}$ particle deposition in a branching airway model. Inhal Toxicol, 2006, 18: 761-771

12 Zhang Z, Kleinstreuer C, Kim C S. Comparison of analytical and CFD models with regard to micron particle deposition in a human 16-generation tracheobronchial airway model. J Aerosol Sci, 2009, 40: 16-28

13 Jin H H, Fan J R, Zeng M J, et al. Large eddy simulation of inhaled particle deposition within the human upper respiratory tract. J Aerosol Sci, 2007, 38: 257-268

14 Yeh H C, Schum G M. Models of human lung airways and their application to inhaled particle deposition. Bull Math Biol, 1980, 42: 461-480

15 Wilcox D C. Turbulence Modeling for CFD. 2nd ed. La Cañada, CA: DCW Industries Inc, 1998

16 Clift R, Grace J R, Weber M E. Bubbles, Drops and Particles. New York: Academic Press, 1978

17 Balashazy I, Hofmann W, Martonen T B. Inertial impaction and gravitational deposition of aerosols in curved tube and airway bifurcations. Aerosol Sci Tech, 1990, 13: 308-321

18 Chan T L, Lippmann M. Experimental measurements and empirical modeling of the regional deposition of inhaled particles in humans. Am Ind Hyg Assoc J, 1980, 41: 399-409

19 Asgharian B, Price O T. Airflow distribution in the human lung and its influence on particle deposition. Inhal Toxicol, 2006, 18: 795-801

20 Subramaniam R P, Asgharian B, Freijer J I, et al. Analysis of lobar differences in particle deposition in the human lung. Inhal Toxicol, 2003, 15: 1-21

Open Access This article is distributed under the terms of the Creative Commons Attribution License which permits any use, distribution, and reproduction in any medium, provided the original author(s) and source are credited. 